\title{
PEG-400 Partitioning in the HCCD/PEG Process for Cs and Sr Recovery
}

\section{$15^{\text {th }}$ Symposium on Separation Science \& Technology for Energy Applications}

The INL is a

U.S. Department of Energy

National Laboratory

operated by

Battelle Energy Alliance

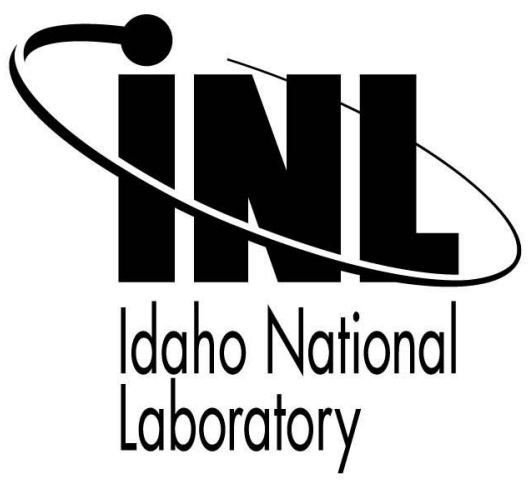

July 2008

This is a preprint of a paper intended for publication in a journal or proceedings. Since changes may be made before publication, this preprint should not be cited or reproduced without permission of the author. This document was prepared as an account of work sponsored by an agency of the United States Government. Neither the United States Government nor any agency thereof, or any of their employees, makes any warranty, expressed or implied, or assumes any legal liability or responsibility for any third party's use, or the results of such use, of any information, apparatus, product or process disclosed in this report, or represents that its use by such third party would not infringe privately owned rights. The views expressed in this paper are not necessarily those of the United States Government or the sponsoring agency. 


\title{
PEG-400 Partitioning in the HCCD/PEG Process for Cs and Sr Recovery
}

\author{
R. S. Herbst, ${ }^{1}$ D. R. Peterman, ${ }^{1}$ and Troy A. Robinson ${ }^{2}$ \\ 'Idaho National Laboratory, Idaho Falls, ID, USA \\ ${ }^{2}$ University of Nevada Las Vegas, Las Vegas, NV, USA
}

\begin{abstract}
The properties of the chloro-protected cobalt bis(dicarbollide) anion in the acidic form (HCCD), and in the presence of polyethylene glycol (PEG400), are well known for the recovery of $\mathrm{Cs}$ and $\mathrm{Sr}$ from acidic radioactive streams. In the early development of HCCD/PEG extraction processes, questions were raised regarding the ability to control the concentration of PEG-400 in the organic phase due to its high solubility in the aqueous process solutions relative to HCCD or the diluent. The purpose of this study was to quantify the partitioning behavior of PEG-400 under a wide variety of relevant process conditions. PEG distribution ratios $\left(\mathrm{D}_{P E G}\right)$ were measured by equilibrium batch contacts between the organic and aqueous phases over a wide range of conditions using radiometric techniques with ${ }^{14} \mathrm{C}$ labeled PEG-400 to monitor the behavior of the bulk material. The results vary dramatically from $0.1<\mathrm{D}_{\mathrm{PEG}}<50$, indicate that the PEG phase transfer kinetics are rapid, and that the aqueous phase nitric acid concentration has minimal impact on PEG solubility. The molar concentration ratio of [HCCD]:[PEG] in the organic phase has the greatest impact on PEG solubility. This ratio should be maintained at [HCCD]:[PEG] greater than or equal to approximately 6 to minimize PEG losses from the organic phase.
\end{abstract}

Keywords: Cesium removal, chlorinated cobalt dicarbollide, PEG-400, radionuclide separations, solvent extraction, strontium removal

\section{INTRODUCTION}

The properties of the chloro-protected cobalt bis(dicarbollide) anion in the protonated or acid form (HCCD), in the presence of polyethylene

Address correspondence to R. Scott Herbst, Idaho National Laboratory, P.O. Box 1625, Idaho Falls, ID, 83415-2208, USA. Fax: (208) 526-8541; E-mail: r.herbst@inl.gov 


\section{R. S. Herbst et al.}

glycol (with an average molecular weight of 400, PEG-400), dissolved in a polar, organic diluent, has been known and studied for three decades for the recovery of $\mathrm{Cs}$ and $\mathrm{Sr}$ from acidic radioactive streams. Pioneering efforts on dicarbollide extraction and chemistry were initially performed by the Nuclear Research Institute and the Institute of Inorganic Chemistry, ̌̌ež, Czech Republic. Later, numerous collaborative efforts further enhanced the understanding and development of HCCD with synergistic additives for the simultaneous, efficient recovery $\mathrm{Cs}$ and $\mathrm{Sr}$ in nuclear applications. Most of these development efforts are described and reviewed in the recent literature (1).

For over a decade, the Khlopin Radium Institute (KRI, St. Petersburg, Russia) and the Idaho National Laboratory (INL, Idaho Falls, Idaho, USA) collaborated on expanding the use of HCCD, in the presence of appropriate synergists, for the recovery of major radionuclides from acidic radioactive waste streams (2). This collaboration culminated with the development of the Universal Extraction (UNEX) process for simultaneous extraction of cesium, strontium, actinides (An), and lanthanides ( $\mathrm{Ln}$ ) from acidic solutions. The UNEX process typically utilizes a solvent consisting of $0.08 \mathrm{M} \mathrm{HCCD}, 0.007-0.02 \mathrm{M}$ PEG-400, and $0.02 \mathrm{M}$ diphenyl-N,N-di-n-butylcarbamoylmethylphosphine oxide (CMPO) in the diluent phenyltrifluoromethyl sulfone $\left(\mathrm{C}_{6} \mathrm{H}_{5} \mathrm{SO}_{2} \mathrm{CF}_{3}\right.$, designated FS-13) and provides simultaneous extraction of all major radionuclides (Cs, $\mathrm{Sr}$, An, and $\mathrm{Ln}$ ) from aqueous $\mathrm{HNO}_{3}$ solutions containing up to $\sim 2$ $\mathrm{M} \mathrm{HNO}_{3}$ and $\sim 4 \mathrm{M}$ total nitrate. The process was initially developed for the treatment of acidic aqueous radioactive wastes that contain relatively low concentrations of the radionuclides, to minimize the volume of material requiring long-term geological disposal $(3,4)$. Much of this early work with the UNEX process is directly relevant to understanding and implementing the HCCD/PEG process (UNEX without the CMPO for An/Ln extraction) for Cs and Sr recovery from spent nuclear fuel.

In the early development of the UNEX process, a question was raised regarding the ability to control the concentration of PEG-400 in the solvent. The PEG- 400 has the greatest solubility in the aqueous process solutions relative to the other components in the UNEX solvent. It is possible to engineer around PEG losses from the organic phase by adding PEG-400 to one of the aqueous feed solutions; this concept was tested for the UNEX process by adding $500 \mathrm{mg} / \mathrm{L}$ PEG-400 to the strip solution (4). The results (based on $\mathrm{Sr}$ distributions and recovery over the course of the test) indicated that this was an effective means of controlling PEG concentration in the organic phase. Despite the known solubility of PEG in process solutions, the amount of PEG washed out of the organic phase has never been quantified. The [HCCD]:[PEG] ratio of $\sim 5: 1$ used in the organic phase was based on the work of Romanovskiy et. al. 
PEG-400 Partitioning in the HCCD/PEG Process

performed during the development of the UNEX process (5). Analysis of earlier data of Rais et. al. indicates that the ratio of [HCCD]:[PEG] should be maintained between 3 and 10 to minimize PEG-400 losses from the organic phase (6).

Previous experimental work indicates that PEG exists in the HCCD/FS-13 organic phase as the protonated, hydrated complex PEG.HCCD $2 \mathrm{H}_{2} \mathrm{O}$ in the presence of HCCD (7). Thus, the proposed equilibria involved with PEG solubility in the organic phase can be described as follows:

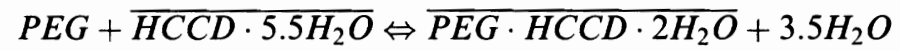

where over bars indicate organic phase species. It is precisely this protonation of PEG by HCCD that keeps the lion's share of the PEG in the organic phase (PEG-400 is miscible in water in all proportions), which, as indicated previously, requires approximately a five-fold excess of HCCD ([HCCD]:[PEG] $\geq \sim 5$ ). The fact that HCCD is a relatively strong (dissociated) acid in the organic phase and that polar organic diluents, i.e., FS-13, can support the additional waters of hydration $\left(5.5\right.$ moles of $\mathrm{H}_{2} \mathrm{O}$ per mole of HCCD) lends credibility to this proposed mechanism (7).

Recent experimental efforts in our laboratory have focused on understanding the fundamental aspects of $\mathrm{Cs}$ and $\mathrm{Sr}$ extraction by the HCCD/PEG-400 system in FS-13. The extraction mechanism (equilibrium reactions) and fundamental thermodynamics of the system are being studied via classical log-log (slope) and van't Hoff analysis methods, as well as by using biphasic calorimetry. Knowledge of the organic phase equilibrium PEG concentration is mandatory for analysis of Sr extraction data obtained by these methods. Due to the lack of quantitative PEG solubility data or analysis methods, the only possible a priori assumption was that PEG was insoluble in the aqueous phase if the molar concentration ratio of $[\mathrm{HCCD}]:[\mathrm{PEG}]$ was maintained at $\sim 5$. Previous experience makes the validity of this assumption highly suspect. The primary objective of this study was to determine the distribution ratio of PEG400 under a variety of relevant conditions and quantify PEG-400 losses from the HCCD/PEG-400/FS-13 extraction system. This knowledge is particularly salient in batch contact experiments for determining the fundamental extraction behavior and thermodynamic properties, e.g., the determination of equilibrium PEG concentration in an organic phase following successive batch-wise pre-equilibrations of the organic with fresh aliquots of aqueous phases. Although specifically targeted to examine equilibrium PEG concentrations during batch contact experiments, this information will likely be relevant or useful in the development of processing schemes based on the HCCD/PEG/FS-13 extraction system. 
R. S. Herbst et al.

\section{EXPERIMENTAL}

The Cs salt of chloro-protected cobalt bis(dicarbollide), was obtained from Katchem (řež, Czech Republic) and used as received without additional purification. A concentrated stock solution of CCD was first prepared by dissolution of the appropriate amount of CsCCD salt in FS-13. This stock solution was converted to HCCD by repeated (4 to 5 ) batch contacts with equal volumes of $4 \mathrm{M} \mathrm{HClO}_{4}$. This stock solution, nominally at $0.25 \mathrm{M}$ HCCD, is diluted and used in subsequent extraction experiments. The HCCD concentration in the organic phase is determined indirectly by potentiometric titration of HCCD with standard base assuming a $1: 1 \mathrm{H}^{+}: \mathrm{CCD}^{-}$complex is formed. The FS-13 was obtained from Marshallton Research Laboratories Inc. (King, NC) and used as received. Other chemicals were of reagent grade purity and used without additional purification.

Carbon-14 labeled PEG-400 $\left({ }^{14} \mathrm{C}\right.$ PEG) was obtained from American Radiolabeled Chemicals, Inc. (St. Louis, MO) as a 3\% aqueous ethanol solution containing $0.1 \mathrm{mCi}{ }^{14} \mathrm{C} / \mathrm{mL}$. From this material, a stock solution of ${ }^{14} \mathrm{C}$ labeled PEG-400 was prepared in $1 \mathrm{M} \mathrm{HNO}_{3}$ with an activity of $0.52 \mu \mathrm{Ci} / \mathrm{mL}(19,100 \mathrm{bq} / \mathrm{mL})$ that was used as the spike solution to introduce the radiolabeled PEG into phases containing quantities of stable

(unlabeled) PEG at various bulk concentrations. Assuming the ${ }^{14} \mathrm{C}$ labeling efficiency is 1 atom of ${ }^{14} \mathrm{C}$ per PEG molecule, the maximum concentration of radiolabeled PEG added into the samples was on the order of $10^{-6} \mathrm{M}^{14} \mathrm{C}$ labeled PEG, indicating the spike addition did not measurably alter the bulk PEG concentration.

Prior to experimental determination of PEG distribution ratios, it was necessary to develop and validate the liquid scintillation counting techniques that were to be used. A NIST traceable ${ }^{14} \mathrm{C}$ standard was obtained from Isotope Products Laboratories (Burbank, CA) and contained $103.9 \mathrm{uCi}{ }^{14} \mathrm{C}$ in $\sim 5 \mathrm{~g}$ of solution. This reference standard was used to develop quench curves and determine detector efficiencies for the composition range of organic and aqueous solutions to be used in the experimental work. Based on these studies, the relative counting errors in liquid scintillation analyses where maintained at or below $\pm 2 \%$.

Distribution ratios $\left(\mathrm{D}_{P E G}=[\mathrm{PEG}]_{\text {org }} /[\mathrm{PEG}]_{a q}\right)$ were measured by equilibrium batch contacts between the organic and aqueous phases at an organic-to-aqueous phase ratio of unity $\left(V_{r}=1\right)$. The two phases were contacted by vortex mixing for several minutes and separated by centrifugation. Each equilibrated phase was sampled and the PEG concentrations determined by liquid scintillation counting (Perkin Elmer Tri-Carb $3170 \mathrm{TR} / \mathrm{SL}$ ). The counting results were used to calculate PEG distribution ratios. Note that the reported PEG concentrations are assumed 


\section{PEG-400 Partitioning in the HCCD/PEG Process}

those of the bulk, unlabelled PEG-400 and not the ${ }^{14} \mathrm{C}$ PEG contained in the system. The partitioning behavior of the bulk PEG was assumed represented by the behavior of radiometrically measured ${ }^{14} \mathrm{C}$ labeled tracer, e.g., that the behavior of the labeled and unlabeled PEG is identical. The assumption may be tested in the future, but does not represent a great leap of faith since the solubility of PEG-200 through PEG-600 are virtually identical; all are miscible with water in all proportions.

\section{RESULTS AND DISCUSSION}

\section{Nomenclature}

Using the experimental methods previously defined, the total equilibrium concentrations of PEG-400 were measured for a number of organic and aqueous phases to elucidate the partitioning behavior of PEG in the HCCD/PEG-400/FS-13 solvent extraction system. The concentration ratio of PEG in the organic and aqueous phases at equilibrium is the distribution ratio:

$$
\mathrm{D}_{\mathrm{PEG}}=[\mathrm{PEG}]_{\text {org }} /[\mathrm{PEG}]_{a q}
$$

Distribution ratios for PEG were measured under a wide range of experimental conditions and vary dramatically from $0.1>D_{\mathrm{PEG}}>50$. Since such a wide range of PEG-400 D's are to be compared in the ensuing discussion, it is convenient to discuss the results in terms of the fractional amounts of PEG in each phase after a single equilibration contact. Thus, the following terms are defined:

$$
\begin{aligned}
& p \equiv \text { fraction of PEG in the organic phase after equilibration } \\
& =\mathrm{D}_{\mathrm{PEG}} \mathrm{V}_{\mathrm{r}} /\left(\mathrm{D}_{\mathrm{PEG}} \mathrm{V}_{\mathrm{r}}+1\right) \\
& q \equiv \text { fraction of PEG in the aqueous phase after equilibration } \\
& =1 /\left(\mathrm{D}_{\mathrm{PEG}} \mathrm{V}_{\mathrm{r}}+1\right)
\end{aligned}
$$

where $V_{r}$ is the organic-to-aqueous phase volume ratio:

$$
\mathrm{V}_{\mathrm{r}}=\mathrm{V}_{\text {org }} / \mathrm{V}_{a q}
$$

Note that all equilibration contacts were performed at a phase volume ratio of $\mathrm{V}_{\mathrm{r}}=1$, and

$$
\begin{gathered}
p=\mathrm{D}_{\mathrm{PEG}} /\left(\mathrm{D}_{\mathrm{PEG}}+1\right) \\
q=1 /\left(\mathrm{D}_{\mathrm{PEG}}+1\right)
\end{gathered}
$$




\section{R. S. Herbst et al.}

Of course:

$$
p+q=1
$$

\section{Kinetics of PEG Extraction}

Typically, the kinetics of metal ion transfer between immiscible phases is relatively rapid and mixing times of a few minutes or less are sufficient to achieve equilibrium. It is of practical importance to determine if rapid kinetics is also the case with regard to PEG extraction. An initial experiment was performed to determine the effect of phase mixing time on $D_{\mathrm{PEG}}$. The organic phase was $0.08 \mathrm{M}$ HCCD $+0.016 \mathrm{M}$ PEG-400 in FS-13 spiked with ${ }^{14} \mathrm{C}$ PEG, the aqueous phase was $1 \mathrm{M} \mathrm{HNO}_{3}$. The test was conducted with an organic-to-aqueous phase ratio of $\mathrm{V}_{r}=1$ and samples were centrifuged for 2 minutes to separate the phases. The results are presented in Fig. 1. These data indicate that the distribution of PEG-400 between the organic and aqueous phases is kinetically rapid $\left(<2.7 \%\right.$ change in $D_{P E G}$ for all contact times) and equilibrium is reached within 30 seconds of vortex mixing. A mixing time of at least 2 minutes was used in all subsequent batch contact experiments to insure that equilibrium was obtained.

\section{PEG Partitioning in the Absence of HCCD}

As previously mentioned, PEG-400 is completely miscible in water in all proportions and the presence of HCCD in the organic phase is required

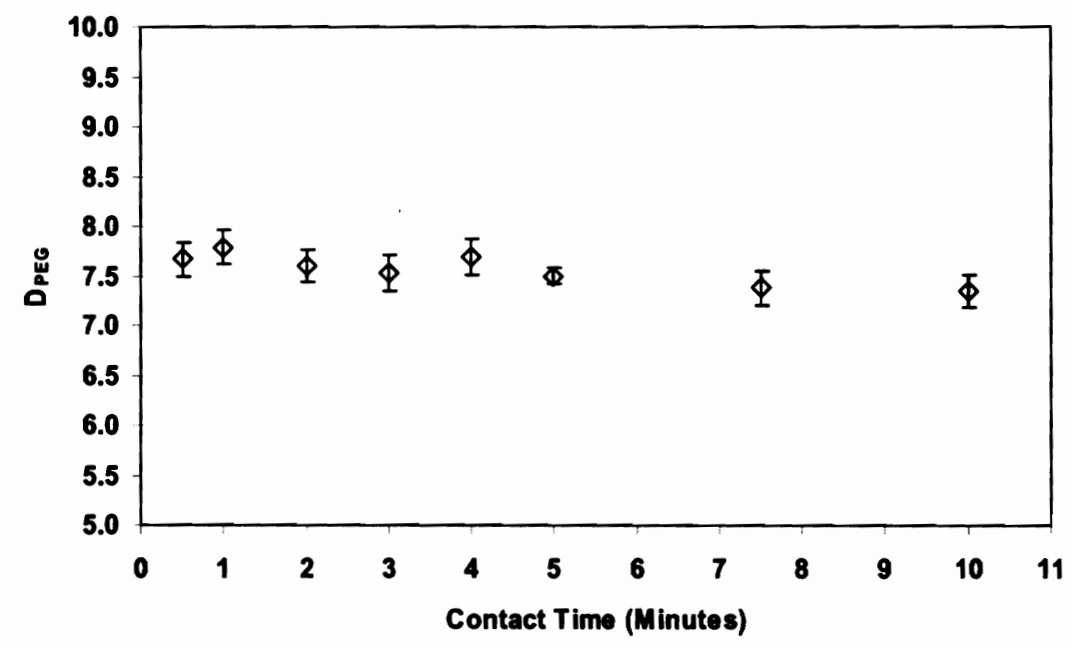

Figure 1. Results of PEG phase transfer kinetic testing. 
PEG-400 Partitioning in the HCCD/PEG Process

to limit PEG losses to the aqueous phase. It is of interest to quantitatively examine the partitioning of PEG between the FS-13 organic diluent and a nitric acid aqueous phase, in the absence of HCCD. A 0.016 M PEG-400 solution in FS-13 was prepared and spiked with ${ }^{14} \mathrm{C}$ labeled PEG. This organic was contacted three consecutive times with fresh aqueous solutions containing $0.5 \mathrm{M}, 1 \mathrm{M}$, or $3 \mathrm{M} \mathrm{HNO}_{3}$ at an organic to aqueous phase ratio of $\mathrm{V}_{\mathrm{r}}=1$. The results of these experiments are indicated in Table 1. Noteworthy is the fact that approximately $95 \%$ of the PEG400 is extracted from the FS-13 in the first contact. Of the remaining PEG in the organic phase, an additional $\sim 13 \%$ is extracted from the organic during the second extraction contact with fresh aqueous, and an additional $\sim 7 \%$ of the remaining PEG is extracted in the third contact. These data indicate that after three successive contacts with aliquots of fresh nitric acid solution, a total of $\sim 96 \%$ of the PEG-400 initially present in the organic phase has been extracted from the FS-13 organic phase. Furthermore, PEG washout from FS-13 appears to be virtually independent of the concentration of $\mathrm{HNO}_{3}$ used in the aqueous phase. These data verify that in the absence of HCCD, PEG losses from the FS-13 organic phase are rather high.

\section{Effect of $\mathrm{HNO}_{3}$ and HCCD on PEG Partitioning}

The results presented in Table 1 indicate that the aqueous concentration of $\mathrm{HNO}_{3}$ has minimal impact on $\mathrm{D}_{\mathrm{PEG}}$, at least in the absence of HCCD. The effect of $\mathrm{HNO}_{3}$ concentration on PEG partitioning was further explored, except this time in the presence of HCCD. Data regarding

Table 1. PEG partitioning results in the absence of HCCD

\begin{tabular}{lccccc}
\hline & $\begin{array}{c}\text { Contact } \\
\#\end{array}$ & $\begin{array}{c}\text { \%EG }]_{\text {organic }} \\
(M)\end{array}$ & $\begin{array}{c}\text { Initial PEG } \\
\text { remaining in } \\
\text { organic phase }\end{array}$ & $\mathrm{D}_{\text {PEG }}$ & $\begin{array}{c}\mathrm{q}=1 / \\
\left(\mathrm{D}_{\mathrm{PEG}}+1\right)\end{array}$ \\
\hline 0.5 & 1 & 0.0009 & $5.5 \%$ & 0.06 & 0.95 \\
0.5 & 2 & 0.0008 & $4.8 \%$ & 6.9 & 0.13 \\
0.5 & 3 & 0.0007 & $4.6 \%$ & 13.6 & 0.07 \\
1 & 1 & 0.0009 & $5.4 \%$ & 0.06 & 0.94 \\
1 & 2 & 0.0008 & $4.8 \%$ & 7.3 & 0.12 \\
1 & 3 & 0.0007 & $4.6 \%$ & 14.5 & 0.06 \\
3 & 1 & 0.0008 & $5.2 \%$ & 0.06 & 0.95 \\
3 & 2 & 0.0007 & $4.6 \%$ & 6.0 & 0.14 \\
3 & 3 & 0.0007 & $4.4 \%$ & 10.9 & 0.08 \\
\hline
\end{tabular}


R. S. Herbst et al.

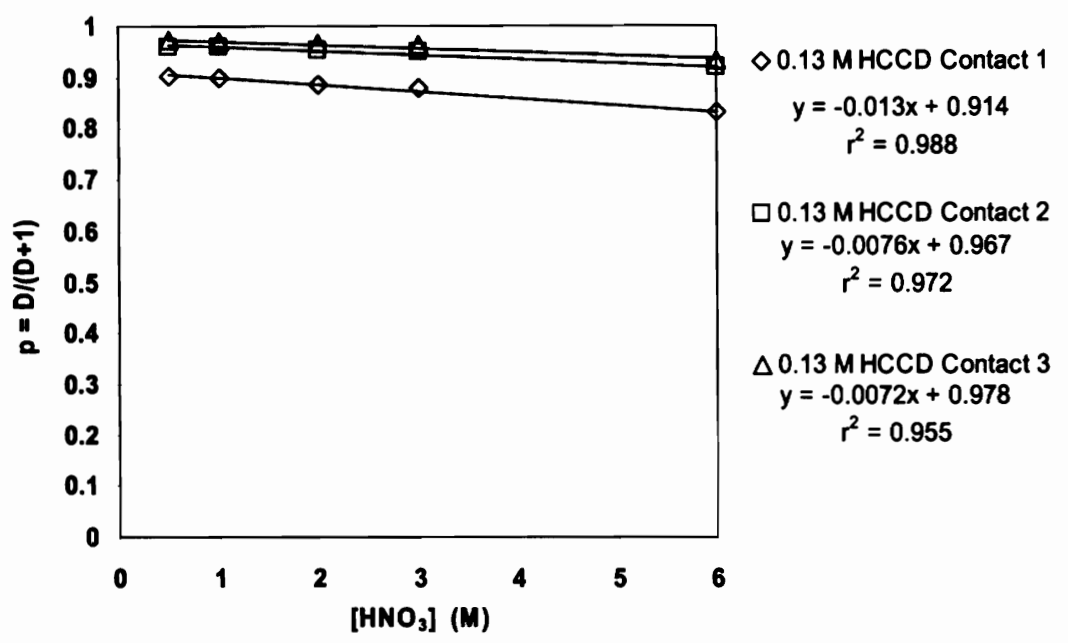

Figure 2. Effect of $\mathrm{HNO}_{3}$ on $p$ for an initial ratio of $[\mathrm{HCCD}]:[\mathrm{PEG}]=4.8$.

the fraction of PEG in the organic phase at equilibrium, $p$, as a function of aqueous $\mathrm{HNO}_{3}$ concentration for organic phases containing $0.13 \mathrm{M}$ HCCD + 0.027 M PEG-400 and 0.08 M HCCD + 0.016 M PEG-400 are indicated in Figs. 2 and 3, respectively. Note that there were three successive contracts of each organic phase with fresh aliquots of the respective $\mathrm{HNO}_{3}$ solutions. There is a slight negative dependence of $p$ on $\mathrm{HNO}_{3}$ concentration with both data sets. The results of linear

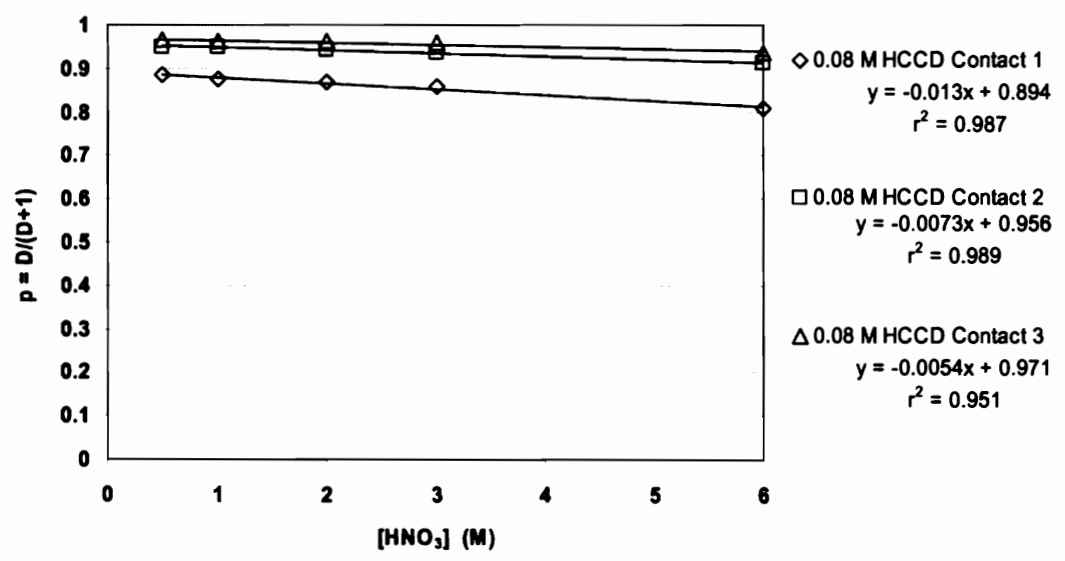

Figure 3. Effect of $\mathrm{HNO}_{3}$ on $p$ for an initial ratio of $[\mathrm{HCCD}]:[\mathrm{PEG}]=5.0$. 


\section{PEG-400 Partitioning in the HCCD/PEG Process}

regression analysis for the each different contact is also indicated in the figure captions, indicating good linearity and the very slight negative dependence (slopes $<-0.013$ ) of $p$ with increasing $\mathrm{HNO}_{3}$ concentration. These data indicate that the aqueous phase nitric concentration is not a predominate factor in the partitioning of PEG between the two phases at these experimental conditions. More importantly, in contrast to the previous results where HCCD was absent from the organic phase, greater than $80 \%$ of the PEG was retained in the organic phase when HCCD was included in the organic phase.

\section{Effect of the [HCCD]:[PEG] Ratio}

Another interesting observation associated with the data in Figs. 2 and 3 is that with successive contacts at a fixed $\mathrm{HNO}_{3}$ concentration, $p$ appears to increase toward some relatively constant value. This is to be attributed to incremental increases in the PEG distribution ratio (note that in the limit as $D_{P E G}$ becomes infinite, $p$ tends to a value of unity). On the other hand, it is indicated that the concentration of HCCD obviously plays an important role in the value of $D_{\mathrm{PEG}}$ and therefore $p$. It is of interest to further examine this effect and the final (equilibrium) molar concentration ratio of [HCCD]:[PEG] in the organic phase is shown as a function of contact number in Fig. 4. The data previously presented for three consecutive contacts with $1 \mathrm{M} \mathrm{HNO}$ for $0.13 \mathrm{M} \mathrm{HCCD}$ and $0.08 \mathrm{M}$ HCCD (taken from Figs. 2 and 3, respectively) are indicated. Additionally, a series of six consecutive equilibrium batch contacts between fresh

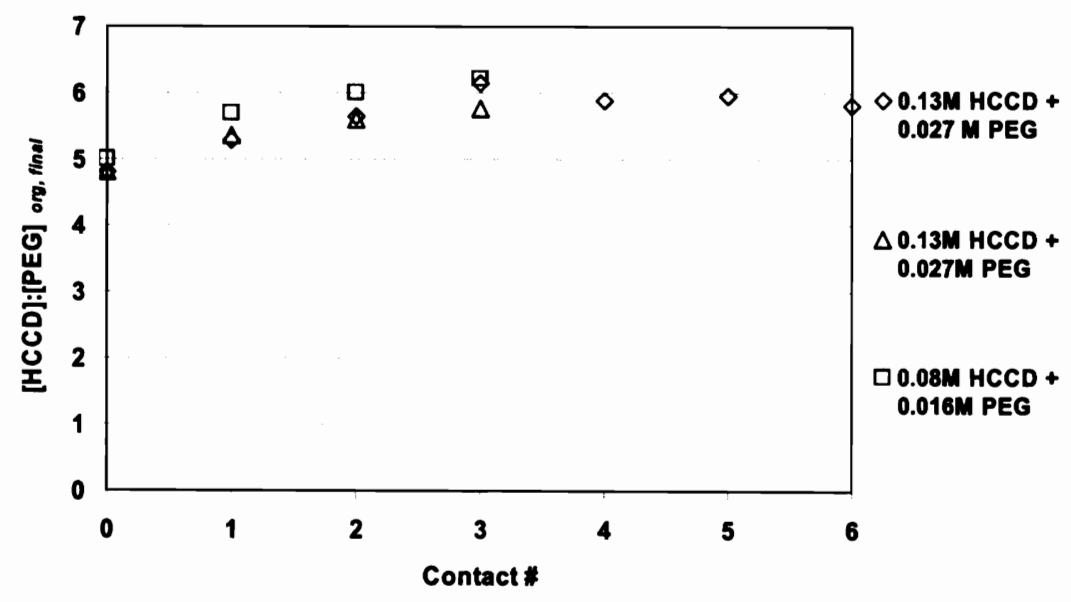

Figure 4. Equilibrium molar concentration ratio of [HCCD]:[PEG]. 


\section{R. S. Herbst et al.}

aqueous aliquots of $1 \mathrm{M} \mathrm{HNO}_{3}$ and an organic phase initially composed of $0.13 \mathrm{M} \mathrm{HCCD}+0.027 \mathrm{M}$ PEG in FS-13 are also indicated in Fig. 4. It is apparent that the concentration ratio of [HCCD]:[PEG] is tending toward a value of 6 as the number of contacts increases.

The data in Fig. 4 emphasizes the importance of the [HCCD]:[PEG] molar concentration ratio, and it is of interest to examine the effects that the molar concentration ratio of [HCCD]:[PEG] present in the system has on the quantity $p$ after a single equilibrium contact. Figure 5 indicates the measured value of $p$ for a range of different [HCCD]:[PEG] ratios. Note that the compendium of data indicated in Fig. 5 represents a variety of different experimental conditions, where the concentration of HCCD was either $0,0.08 \mathrm{M}$ or $0.13 \mathrm{M}$ and the PEG concentration varied between $0.0008 \mathrm{M}$ and $0.13 \mathrm{M}$. The nitric acid concentration in the aqueous phase was varied from 0.5 to $6 \mathrm{M} \mathrm{HNO}_{3}$. The results in Fig. 5 indicate that in the region of [HCCD]:[PEG] $<\sim 5$, there is a definite knee in the data, below which PEG is substantially washed out of the organic phase as the concentration ratio of [HCCD]:[PEG] tends toward zero. The data in Fig. 5 is consistent with those previously indicated, and strongly suggest that the optimal ratio of [HCCD]:[PEG] in the organic phase should be increased from the present nominal value of $\sim 5$, as determined in the early developmental work $(4,5)$, to a minimum ratio of $[\mathrm{HCCD}]:[\mathrm{PEG}] \geq 6$, and perhaps even slightly higher, in order to minimize PEG losses from the organic phase. Ultimately, such a change in the organic phase composition will require additional testing to insure there

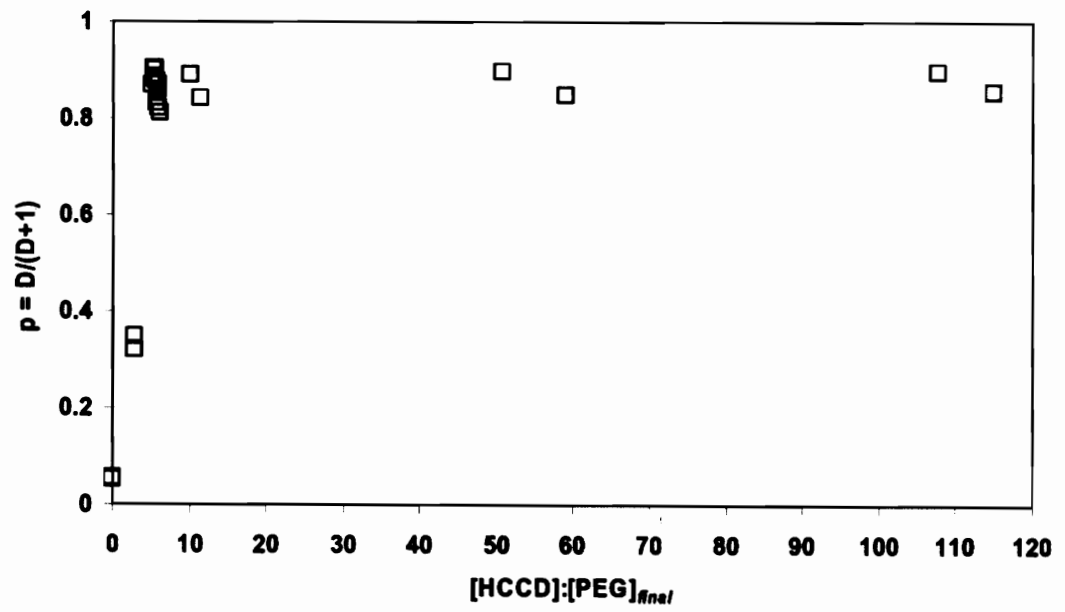

Figure 5. Scatter diagram of $p$ versus the molar ratio of [HCCD]:[PEG]. 


\section{PEG-400 Partitioning in the HCCD/PEG Process}

are no significant impacts on the Cs and $\mathrm{Sr}$ D's in the HCCD/PEG process.

\section{Limiting [HCCD]:[PEG] Ratio}

It is also of interest to evaluate $D_{P E G}$ under limiting conditions where the concentration ratio of [HCCD]:[PEG] becomes extremely large, i.e., tends to infinity, to insure that the observed value remains essentially constant. In order to evaluate the effects of the limiting [HCCD]:[PEG] ratio, $\mathrm{D}_{\text {PEG }}$ was measured at three aqueous nitric acid concentrations ( $1 \mathrm{M}, 3 \mathrm{M}$, and $6 \mathrm{M} \mathrm{HNO}_{3}$ ) for an organic solution of $0.11 \mathrm{M} \mathrm{HCCD}$ in FS-13 (thrice pre-equilibrated with the respective $\mathrm{HNO}_{3}$ containing the aqueous phase). Following the $\mathrm{HNO}_{3}$ pre-equilibration, ${ }^{14} \mathrm{C}$ labeled $\mathrm{PEG}$ was spiked into the organic phase which was then contacted with a solution of fresh $\mathrm{HNO}_{3}$ and the PEG distribution determined. Stable PEG-400 was purposely not added to the system in order to maintain the ratio of [HCCD]:[PEG] at an experimental maximum. The approximate ratio of $[\mathrm{HCCD}]:[\mathrm{PEG}] \approx 1.5 \mathrm{E} 4$ was estimated in the initial organic phase, based on the previously mentioned ${ }^{14} \mathrm{C}$ labeling assumption of the PEG tracer solution. The results of these experiments, shown in Table 2, verify several aspects of the previous discussions. First, the measured values of $p=\sim 0.9$ at a very high [HCCD]:[PEG] ratio (i.e., approaches infinite) are consistent with the results in Fig. 5, indicating a limiting value of $p$ between 0.85 and 0.9 . Finally, the concentration of $\mathrm{HNO}_{3}$ has minimal impact on $p$. Note that for the data presented in Table 2, three independent measurements were performed at each respective $\mathrm{HNO}_{3}$ concentration. Thus, the average and standard deviations of these independent measurements are presented.

\section{Estimation of the Final Organic Phase PEG Concentration after $\mathbf{n}$ Equilibration Contacts}

Experimental efforts in our laboratory have focused on understanding the fundamental aspects of $\mathrm{Cs}$ and $\mathrm{Sr}$ extraction by the HCCD/PEG-400

Table 2. Results of PEG partitioning at a limiting [HCCD]:[PEG] ratio

\begin{tabular}{lcc}
\hline$\left[\mathrm{HNO}_{3}\right], \mathrm{M}$ & Average $\mathrm{D}_{\mathrm{PEG}}$ & $\mathrm{p}=\mathrm{D}_{\mathrm{PEG}} /\left(\mathrm{D}_{\mathrm{PEG}}+1\right)$ \\
\hline 1 & $13.4 \pm 0.42$ & $0.931 \pm 0.002$ \\
3 & $8.97 \pm 0.21$ & $0.900 \pm 0.002$ \\
6 & $6.67 \pm 0.36$ & $0.869 \pm 0.006$ \\
\hline
\end{tabular}




\section{R. S. Herbst et al.}

system in FS-13; knowledge of the organic phase equilibrium PEG concentration is mandatory for the analysis of $\mathrm{Sr}$ extraction data. Much of the $\mathrm{Sr}$ (and Cs) distribution data collected to date have been obtained with nitric acid pre-equilibrated organics with compositions similar (if not identical) to those used in this study $(0.08 \mathrm{M}$ HCCD $/ 0.016 \mathrm{M}$ PEG or $0.13 \mathrm{M}$ HCCD/0.027 M PEG in FS-13). Consequently, it would be useful to have a correlation of the final equilibrium PEG concentration in an organic phase following successive batch-wise pre-equilibrations of the organic with fresh aliquots of aqueous $\left(\mathrm{HNO}_{3}\right)$ phases. It has already been noted that $p$ strongly depends on the molar ratio of [HCCD]:[PEG] in the organic phase, and above a ratio of $\sim 6$ the fractional amount of PEG left in the organic phase remains relatively constant. Using the previously discussed PEG distribution data where the ratio of [HCCD]:[PEG] $>4.8$ results in the linear correlation indicated graphically in Fig. 6. All other data are included: those for the different $\mathrm{HNO}_{3}$ concentrations, for each of $n$ consecutive contacts with fresh aqueous phase, and for the different organic phase HCCD concentrations. The resulting correlation in Fig. 6 indicates very good linearity with a correlation coefficient of $r^{2}=0.998$. Note the intercept was forced through zero in the regression analysis shown in Fig. 6, which introduces negligible error into the correlation

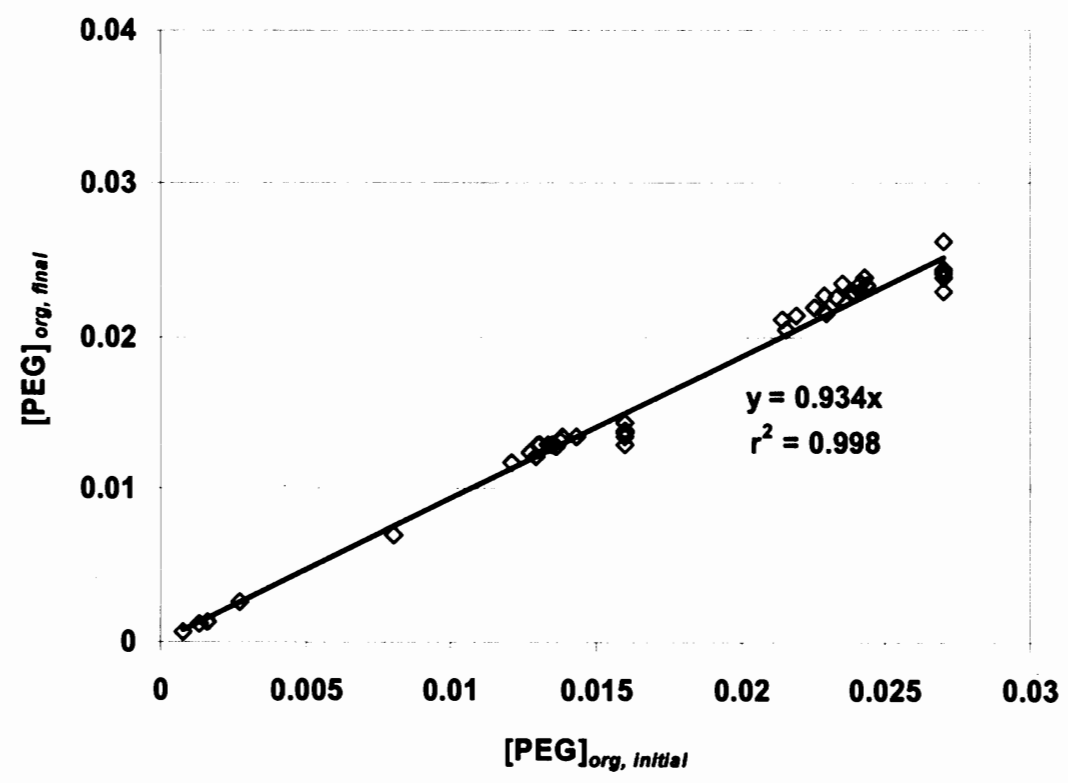

Figure 6. Correlation of the final vs. initial organic phase PEG concentration, $[P E G]$. Experimental data for the initial [HCCD]:[PEG] $>4.8$ only. 


\section{PEG-400 Partitioning in the HCCD/PEG Process}

$\left(y=0.936 x-0.00004, r^{2}=0.983\right.$ when the intercept is not forced through 0 ). The excellent correlation of the experimental data indicates that the fraction of PEG remaining in the organic phase, $p$, is reasonably constant with an average value of $p=0.934$ under these conditions. It should be noted that including those data collected at ratios of [HCCD]:[PEG] $<4.8$ dramatically impacts the fit or linearity of the correlation since those data were collected the region where $p$ is not constant.

Finally, during repeated equilibrations of an initial organic with fresh aliquots of aqueous phase (such as is encountered during organic phase pre-equilibrations for the measurement of $\mathrm{Cs}$ and $\mathrm{Sr}$ distribution ratios), it can be shown (8) that the concentration of PEG in the organic phase after $n$ successive contacts with fresh aliquots of aqueous is given by:

$$
\mathrm{C}_{\text {org, final }}=p^{n} \mathrm{C}_{o}
$$

where $\mathrm{C}_{\boldsymbol{o}}$ is the total or original concentration of PEG in the system prior to any equilibration contacts. This expression is valid only at $V_{r}=1$ and assumes that a constant fractional amount of PEG, $p$, remains in the organic phase with each successive equilibration (as indicated in Fig. 6). Using the value of $p=0.934$ from the correlation of the experimental data presented in Fig. 6, the following relationship is obtained:

$$
\mathrm{C}_{\text {org. } \text { final }}=0.934^{n} \mathrm{C}_{\text {o }}
$$

Equation (3) predicts that $\sim 6 \%$ of the PEG is removed in each successive batch contact with equal volumes of fresh aqueous phase. Equation (3) should be used only in the region of [HCCD]:[PEG] $>\sim 5$ since most of the data used in the development of this expression was collected in this area. It is likely that at higher [HCCD]:[PEG] concentration ratios and certainly at lower ones, equation (3) is invalid. Despite this fact, the utility of equation (3) is that the concentration of PEG remaining in the organic can be predicted after $n$ pre-equilibrations with $\mathrm{HNO}_{3}$ that have typically been performed prior to measurement of Cs and Sr D's. For example, after equilibration of a $0.13 \mathrm{M}$ HCCD/0.027 M PEG organic with 3 equal volumes of fresh $\mathrm{HNO}_{3}$, the final concentration of PEG in the organic phase would be $\mathrm{C}_{\text {org, }}$ final $=0.934^{3}(0.027 \mathrm{M})=$ $0.0220 \mathrm{M}$ PEG, which is in good agreement with the average experimental value of $0.0222 \pm 0.001 \mathrm{M}$ PEG.

\section{CONCLUSIONS AND RECOMMENDATIONS}

The amount of PEG washed out of the organic HCCD/PEG-400/FS-13 process solvent has been studied using radiometric techniques with ${ }^{14} \mathrm{C}$ labeled PEG-400 to monitor the behavior of the bulk material. The 


\section{R. S. Herbst et al.}

preliminary data indicate that the concentration ratio of [HCCD]:[PEG] has a dramatic effect on PEG solubility in the organic phase. It is recommended that the [HCCD]:[PEG] molar ratio in the organic phase be increased from the presently accepted value of $\sim 5$ to an initial value of $\sim 6$ to 10 to help minimize PEG losses from the organic phase. Data generated to date indicate that the concentration of $\mathrm{HNO}_{3}$ in the aqueous phase has a minimal impact on PEG partitioning. Finally, for the massive quantities of $\mathrm{Sr}$ and $\mathrm{Cs}$ distribution data previously generated with solvent compositions where the [HCCD]:[PEG] ratio was 4.8 or 5 (0.13 M HCCD/0.027 M PEG or 0.08 M HCCD/0.016 M PEG, respectively) the final organic phase PEG concentration after $n$ preequilibration batch contacts with equal volumes of fresh nitric acid can be predicted by the linear expression:

$$
\mathrm{C}_{\text {org. } \text {. } \text { inal }}=0.934^{n} \mathrm{C}_{\mathrm{o}}
$$

where $\mathrm{C}_{\boldsymbol{o}}$ is the original total PEG concentration in the organic phase prior to any contact with an aqueous phase. Note that the expression is valid only in the region where the concentration ratio [HCCD]:[PEG] $\sim 5$ and at an organic-to-aqueous phase ratio of $\mathrm{V}_{r}=1$.

Additional work will be required to better understand PEG solubility and fully optimize the composition of the process solvent. It would be of interest to investigate the effect of temperature on PEG partitioning since phase miscibility is often strongly influenced by temperature. Another area of interest would be on the effects of Sr (and perhaps Cs) loading of the HCCD/PEG solvent on PEG partitioning, since the Sr-PEG complex is perhaps even less miscible in the aqueous phase than the HCCDPEG complex. Finally, additional work is recommended to evaluate PEG losses under $\mathrm{Cs} / \mathrm{Sr}$ back-extraction (stripping) conditions to determine PEG losses in the basic strip solutions.

\section{REFERENCES}

1. Rais, J.; Gruner, B. (2004) Extraction with Metal Bis(dicarbollide) Anions. In: Ion Exchange and Solvent Extraction, Volume 17, Marcus, Y. and SenGupta, A.K., eds.; Marcel Dekker, Inc: New York, NY.

2. Herbst, R.S.; Law, J.D.; Todd, T.A.; Romanovskiy, V.N.; Babain, V.A.; Esimantovski, V.M.; Zaitsev, B.N.; Smirnov, I.V. (2002) Development and testing of a cobalt dicarbollide based solvent extraction process for the separation of cesium and strontium from acidic tank waste. Sep. Sci. Technol., 37 (8): 1807-1831.

3. Herbst, R.S.; Law, J.D.; Todd, T.A.; Romanovskiy, V.N.; Babain, V.A.; Esimantovski, V.M.; Smirnov, I.V.; Zaitsev, B.N. (2002) Universal solvent extraction (UNEX) flowsheet testing for the removal of cesium, strontium, 


\section{PEG-400 Partitioning in the HCCD/PEG Process}

and actinide elements from radioactive, acidic dissolved calcine waste. Solvent Extr. Ion Exch., 20 (4\&5): 429-445.

4. Herbst, R.S.; Law, J.D.; Todd, T.A.; Romanovskiy, V.N.; Smirnov, I.V.; Babain, V.A.; Esimantovski, V.N.; Zaitsev, B.N. (2003) Development of the universal extraction (UNEX) process for the simultaneous recovery of Cs, Sr, and actinides from acidic radioactive waste. Sep. Sci. Technol., 38 (12-13): 2685-2708.

5. Romanovskiy, V.N.; Smirnov, I.V.; Babain, V.A.; Todd, T.A.; Herbst, R.S.; Law, J.D.; Brewer, K.N. (2001) The universal solvent extraction (UNEX) process I: Development of the UNEX process solvent for the separation of cesium, strontium and the actinides from acidic radioactive waste. Solvent Extr. Ion Exch., 19 (1): 1-21.

6. Rais, J.; Sebestova, E.; Selucky, P.; Kyrs, M. (1976) Synergistic effect of polyethylene glycols in extraction of alkaline earth cations by nitrobenzene. J. Inorg. Nucl. Chem., 38: 1742-1744.

7. Stoyanov, E.S.; Smirnov, I.V.; Babain, V.A.; Antov, N.G.; Peterman, D.; Herbst, R.S.; Todd, T.A.; Luther, T.A. (2004) Complexation of polyethylene glycol with $\mathrm{Sr}^{2+}$ and $\mathrm{Ba}^{2+}$ cations in extracts containing chlorinated cobalt dicarbollide. Radiochemistry., 46 (6): 587-592.

8. Peters, D.G.; Hayes, J.M.; Hieftje, G.M. (1974) Chemical Separations and Measurements, Theory and Practice of Analytical Chemistry; W.B. Saunders Co.: Philadelphia, PA. 